\title{
Orthographic facilitation in oral vocabulary acquisition
}

Article

Accepted Version

Ricketts, J., Bishop, D. V.M. and Nation, K. (2009)

Orthographic facilitation in oral vocabulary acquisition.

Quarterly Journal of Experimental Psychology, 62 (10). pp.

1948-1966. ISSN 0272-4987 doi:

https://doi.org/10.1080/17470210802696104 Available at https://centaur.reading.ac.uk/27891/

It is advisable to refer to the publisher's version if you intend to cite from the work. See Guidance on citing.

To link to this article DOI: http://dx.doi.org/10.1080/17470210802696104

Publisher: Psychology Press

All outputs in CentAUR are protected by Intellectual Property Rights law, including copyright law. Copyright and IPR is retained by the creators or other copyright holders. Terms and conditions for use of this material are defined in the End User Agreement.

\section{www.reading.ac.uk/centaur}

\section{CentAUR}

Central Archive at the University of Reading 
Reading's research outputs online 
RUNNING HEAD: ORTHOGRAPHY AND VOCABULARY ACQUISITION

Orthographic facilitation in oral vocabulary acquisition

Jessie Ricketts, DVM Bishop and Kate Nation

University of Oxford

Correspondence:

Jessie Ricketts

Department of Experimental Psychology

University of Oxford

South Parks Road

Oxford, OX1 3UD, UK

Email: jessie.ricketts@psy.ox.ac.uk

Tel: 01865271328 


\begin{abstract}
An experiment investigated whether exposure to orthography facilitates oral vocabulary learning. Fifty-eight typically developing children aged 8-9 years were taught 12 nonwords. Children were trained to associate novel phonological forms with pictures of novel objects. Pictures were used as referents to represent novel word meanings. For half of the nonwords children were additionally exposed to orthography, although they were not alerted to its presence, nor were they instructed to use it. After this training phase a nonword-picture matching post-test was used to assess learning of nonword meaning and a spelling post-test was used to assess learning of nonword orthography. Children showed robust learning for novel spelling patterns after incidental exposure to orthography. Further, we observed stronger learning for nonword-referent pairings trained with orthography. The degree of orthographic facilitation observed in post-tests was related to children's reading levels, with more advanced readers showing more benefit from the presence of orthography.
\end{abstract}

Keywords: reading, orthography, vocabulary, acquisition, learning, development 


\section{ORTHOGRAPHIC FACILITATION IN ORAL VOCABULARY ACQUISITION}

Learning new vocabulary is a life-long endeavour. Children learn an impressive number of words in the first few years of life, and clearly we continue to learn new words throughout adulthood. Once children start school and begin to read, they must learn not only oral vocabulary, but also sight vocabulary. Also, as reading development progresses, vocabulary acquisition can occur via exposure to new words in written as well as oral contexts. In this study we sought to investigate whether presenting children with spellings while they are learning the meanings of new words facilitates oral vocabulary acquisition. Influential approaches to vocabulary instruction do not emphasise the use of spelling patterns while teaching children new words. For example, in a recent book Beck, McKeown and Kucan (2002) recommend that spellings are shown to children but only after words and their meanings have been taught.

Learning new oral vocabulary involves making links between a word's pronunciation (phonology) and its meaning (semantics). For example a child must learn that a dog is an animal that barks, has fur and so on. Learning sight vocabulary involves making additional links between these representations and a word's spelling or orthography i.e., the word ' $d o g$ ' is spelled d-o-g. When we learn sight vocabulary we may map orthography onto a known word (children are likely to know the word $\operatorname{dog}$ when they come to the task of learning to read), sometimes however the word is unfamiliar and we construct a representation linking phonology, semantics and orthography more simultaneously.

In the Lexical Quality Hypothesis, Perfetti and Hart (2002) specify that to learn a new sight word a child or adult must acquire and integrate information about word orthography, phonology and semantics. The main tenet of the Lexical Quality Hypothesis is that having many good quality representations is paramount for effective reading i.e., both quality and quantity are important. The hypothesis remains underspecified in terms of the exact mechanisms that underpin the development of good quality representations. However, Perfetti and Hart suggest that a good quality representation is 
operationalised as the efficient and accurate retrieval of a word's pronunciation, meaning and/or spelling in response to one of the other constituents. So, for reading, a word has a good quality representation if orthography efficiently activates its pronunciation and/or meaning.

In this experiment we used a learning paradigm to investigate whether the presence of orthography boosts oral vocabulary acquisition. In other words, we sought to investigate whether a lexical representation that includes phonology, orthography and semantics (as in sight vocabulary) is of higher quality than a lexical representation that includes only phonological and semantic information (as in oral vocabulary). Perfetti and Hart (2002) propose that once orthography is learnt it can activate semantics directly and therefore phonological representations become redundant for the process of reading. This is consistent with a recent implementation of the PDP connectionist model of reading (Harm \& Seidenberg, 2004) in which increased learning resulted in words being processed via direct links from orthography to semantics, as well as via phonology. This suggests that for a known word (with a good quality representation) orthography and phonology will be interchangeable and meaning will be activated directly from one or the other depending on the demands of the task i.e., phonology for oral language and orthography for reading.

The Lexical Quality Hypothesis suggests that phonological and orthographic representations are inextricably linked for familiar words. Indeed there is much evidence to suggest that a word's orthography affects the speed and accuracy of processing its phonology (e.g., Castles, Holmes, Neath, \& Kinoshita, 2003; Seidenberg \& Tanenhaus, 1979; Ventura, Morais, \& Kolinsky, 2007) and vice versa (Perfetti, Bell, \& Delaney, 1988; Ziegler, Jacobs, \& Klüppel, 2001). For example, Castles et al. (2003) found that both adults and children are susceptible to orthographic intrusions when performing a phoneme deletion task.

Interactions between phonological and orthographic representations could lead to positive as well as negative behavioural effects, with orthographic representations aiding learning and memory for phonological forms (for similar proposals see Ehri, 2005; Hu, 2008; McKague, Davis, Pratt, \& 
Johnston, 2008; Ventura, Morais, Pattamadilok, \& Kolinsky, 2004). Orthographic inputs are less transient across time than phonological inputs, and they are less variable across individuals and contexts. Nelson, Balass and Perfetti (2005) taught adults definitions for rare words in the oral or visual modality. They observed faster learning for printed words than spoken words, demonstrating that adults find it easier to learn links between orthography and semantics than between phonology and semantics. In addition, there are reports of learning/memory advantages for visual stimuli over verbal stimuli in children. In a study with children aged between 7 and 11 years, Hulme, Goetz, Gooch, Adams and Snowling (2007) investigated paired-associate learning for pairings between two aurally presented nonwords, two visually presented abstract shapes or between verbal-visual nonword-shape pairs. They showed that children learned visual-visual pairings between abstract shapes more easily than verbalvisual pairings, which in turn were learned more easily than verbal-verbal pairings. Although children were learning abstract shapes and not orthography (for a study with orthography see Reitsma, 1983), this indicates that children find visual stimuli easier to learn than verbal stimuli and further, pairings between one visual and one verbal stimulus were easier to learn than pairings between two verbal stimuli.

A learning advantage for orthographic/visual stimuli over phonological stimuli is consistent with the proposal that for literate children (and adults), orthography can support learning for more variable and transient phonological forms. Direct evidence for this claim comes from a study conducted by Ehri and Wilce (1979; for a similar study with second language learners see Hu, 2008). In a series of four experiments, children aged 6 to 8 years were taught pronunciations for monosyllabic nonwords. Across all experiments, learning was superior for items trained in conjunction with an appropriate spelling pattern. In addition, more advanced readers showed more benefit from the presence of orthography. Hulme et al. (2007) further specified this relationship between visual-verbal learning and individual differences in reading. Using structural equation modelling they showed that after controlling for a child's phonological skills, learning explained unique variance in exception word 
reading (reading of words with inconsistent spelling-sound mappings such as yacht and blood), but not nonword reading.

Rosenthal and Ehri (2008) recently followed up this research by investigating whether the presence of orthography during training facilitates learning of new word meanings, as well as their pronunciations. They taught two groups of children - aged either 7 to 8 years or 10 to 11 years - from low socio-economic backgrounds the pronunciation and meaning of low-frequency nouns. Although it was unlikely that children knew the phonological form of these rare words, their meanings were all closely related to concepts that children would know. Younger children learned simplified definitions for 12 monosyllabic words (e.g., cur: a homeless dog) and older children learned 20 words with two or three syllables. All words were learned with reference to a picture and for half of the words orthography was also presented underneath the picture.

Rosenthal and Ehri (2008) showed that children in both age groups were more likely to learn the pronunciations, meaning and spellings of words that had been learned with orthography. Thus, as well as supporting the development of phonological representations, their data suggest that orthographic information can aid learning of new word meanings. As in Ehri and Wilce (1979), children with better reading scores showed superior learning. This was the case across all pronunciation and spelling measures; however, it did not hold across all measures tapping learning for meaning, possibly due to ceiling effects. Overall, this suggests that the presence of orthography aids learning of new words, and is most beneficial for children with greater orthographic knowledge.

Our experiment was conducted prior to the publication of Rosenthal and Ehri (2008) and was therefore not designed to replicate their study. Nonetheless, we used different methodology to address the same central question; whether the presence of orthography aids oral vocabulary acquisition in children. We aimed to probe two aspects of vocabulary learning; learning of links between phonology and meaning, and learning of orthographic patterns - processes we term semantic and orthographic learning respectively. We employed a somewhat different paradigm, akin to paired-associate learning, 
and recruited a large group of children aged 8 to 9 years from mixed socio-economic backgrounds. To approximate oral vocabulary learning, children were trained on pairings between orally presented nonwords (phonology) and photographs of novel 3D objects (semantics). In contrast to Rosenthal and Ehri, we used nonwords to ensure that the new 'words' were novel to children. We also used pictures of novel objects to represent meaning so that children were learning new labels for new and unfamiliar objects rather than new labels for known concepts. Children were explicitly told that they were learning the names of 'alien' objects rather than real words. As in Rosenthal and Ehri's study, orthography was presented alongside phonology and semantics for half of the items. Children were not alerted to the presence of orthography, nor were they instructed to use it. After six training trials with each new 'word', semantic learning was assessed using a nonword-picture matching task and orthographic learning was assessed using a spelling task.

In opaque languages such as English, phonemes can be represented by more than one grapheme and vice versa. Children learning these languages must learn to spell words with inconsistent as well as consistent phonology to orthography mappings. To simulate this complexity, we included consistent and inconsistent stimuli. This allowed us to probe orthographic learning in cases where orthography can not be inferred reliably from phonology. According to the self-teaching hypothesis (Share, 1995), successful decoding attempts provide a foundation for orthographic learning to occur. Indeed, a large number of studies have demonstrated that children successfully learn word-specific orthographic representations after reading novel words, both aloud and silently (e.g., Bowey \& Miller, 2007; Cunningham, 2006; Nation, Angell, \& Castles, 2007; Share, 2004). The self-teaching hypothesis suggests that children use knowledge gleaned from previous exposures to a word's orthography to produce its spelling. Alternatively, literate children can use their knowledge of how phonology usually maps onto orthography to spell words directly from phonology. If the word is consistent, either strategy will lead to a correct spelling attempt. In contrast, using phonology-orthography relations to spell an inconsistent word could lead to more than one plausible orthographic pattern e.g., blood could legally 
be spelled b-l-u-d. Therefore, to ensure accuracy children must employ prior knowledge of wordspecific orthography to spell these words. We expected that our participants' experience of formal literacy instruction (approximately five years) would be sufficient to support spelling of our simple consistent nonwords without prior exposure to their orthography. By also including inconsistent nonwords we aimed to investigate whether the children could use limited and incidental experience of word-specific orthography to spell these items correctly.

In sum, our primary aim was to investigate whether the incidental presence of orthography boosts performance in a word learning paradigm. On the basis of the Lexical Quality Hypothesis (Perfetti \& Hart, 2002), we expected that children would benefit from the presence of orthography when learning new words. This hypothesis was strengthened by a recent study (Rosenthal \& Ehri, 2008), which showed that children were more likely to learn the meanings and spelling patterns for words trained with orthography. We also sought to investigate how individual differences in orthographic skill relate to learning. We predicted that more advanced readers would benefit more from orthography when performing our orthographic and semantic tasks (Rosenthal \& Ehri, 2008). More specifically, we anticipated that exception word reading would be more closely related to learning than nonword reading (Hulme et al., 2007).

\section{METHOD}

\section{Participants}

The participants were 58 children (23 boys and 35 girls) aged $8-9$ years $(M$ age $=9.04, S D=$ .28). Children were recruited from seven mainstream schools serving socially varied catchment areas in Oxford, UK and had been exposed to the mixed teaching methods prescribed by the UK National Literacy Strategy. Children spoke English as a first language, and none had any recognised special 
educational needs. The study was approved by the Central University Research Ethics Committee at the University of Oxford.

Materials and procedure

\section{Word learning experiment}

At the beginning of the session the experimenter explained to children that they would be learning "some made up words... words for some new things... they are things that an alien might use" and they were introduced to a picture of an alien. During training children learned 12 pairings between orally presented nonwords and pictures of novel 3D objects. For half of these pairings, nonword orthography was presented above the picture. The consistency of spelling-sound relations was also manipulated. After training, two post-tests assessed learning. Learning for meaning was measured using a nonword-picture matching task and in a second task children were asked to spell nonwords to dictation. All tasks were presented using a computer screen and headphones. Within each task stimulus presentation was controlled by the E-Prime program (Schneider, Eschman, \& Zuccolotto, 2002a, 2002b) which randomised order of presentation and recorded the speed and accuracy of children's responses.

Stimuli

Twelve nonwords containing four or five letters and three or four phonemes (CVC, CVVC or CVCC) were constructed for the experiment (see Appendix A for nonword stimuli). Each child was exposed to six nonwords without orthography (orthography absent condition) and six with orthography (orthography present condition) and this was counterbalanced across participants such that all nonwords appeared in both orthography conditions for approximately the same number of children (as data for two children were not recorded correctly there were 30 children in one group and 28 in the other). 
Each nonword was paired with a 'novel' 3D object, so the same nonword-referent pairs were used for all children. Photos of 3D objects (e.g., unfamiliar tools, an antique telephone, a rare musical instrument) were used as referents. An example of one of the objects (a bell tree) is presented in Appendix B. Pilot testing was conducted with 14 adults to select items that were unfamiliar. Although none of the objects were identified by name, at least one adult identified a function for each object (e.g., a musical instrument for the bell tree presented in Appendix B). Therefore, it was assumed that objects would be novel to children, while also affording some kind of function or use.

Nonwords were constructed for each of three spelling-sound consistency conditions: consistent (C), inconsistent consonant (IC) and inconsistent vowel (IV). Consistency was defined in terms of the number of potential spellings for sounds occurring in monosyllabic words (data taken from the Children's Printed Word Database; Masterson, Dixon \& Stuart, 2002). Four C items were made up of consistent consonants and vowels, where there is a one-to-one relationship between each phoneme and a grapheme. No individual phoneme occupied the initial, medial or final position twice and a double s was used at the end of luss to make this item longer and thus more orthographically similar to the other items. The inconsistent items were constructed in the same way as $\mathrm{C}$ items, except that one phoneme was inconsistent. For the four IC items a consonant that has more than one legal spelling occupied either the initial or terminal position of the nonword. Consonants that can be spelled with a silent letter were used for this purpose and the less frequent spelling (with the silent letter) was adopted (e.g., $/ \mathrm{m} / \rightarrow \mathrm{mb}$ rather than $\mathrm{m})$. The four IV items included an inconsistent vowel in the medial position. According to the Children's Printed Word Database these vowels are spelled using different graphemes with approximately equal frequency. One spelling was selected at random for the experiment (e.g., $/ \mathrm{i} / \rightarrow$ ee rather than ea).

\section{Training}

Before training children were familiarised with the sound pattern for all 12 nonwords. The sounds were presented in a random order one at a time and were repeated until children produced 
correct pronunciations. Following this, there were six training sessions comprising three repetition sessions and three production sessions. Repetition and production sessions were alternated; children completed a repetition session first followed by a production session and so forth to make up six sessions in total. In each session, children were exposed to each item once (in a random order) so by the end of training children had been exposed to each item six times.

During repetition sessions, children were presented with a picture and after a short delay (700 ms) they heard the associated nonword. For items trained with orthography, the spelling additionally appeared above the picture, in black Comic Sans MS font, font size 40. Attention was not drawn to the presence of orthography, and children were not instructed to use it. In both conditions, children were required to repeat the nonword, any incorrect pronunciations were corrected, and the experimenter initiated the next trial. Appendix B presents an example of a nonword-referent pairing in orthography absent and present conditions.

In the production sessions children were presented with a picture and asked to produce the appropriate nonword. At this stage orthography was not presented. Then all children heard the correct pronunciation (irrespective of the accuracy of their response), which was accompanied by the orthography (as above) for orthography present trials.

\section{Post-tests}

Nonword-picture matching. Children were presented with the nonword-picture matching task immediately after training to assess whether they had learned nonword 'meanings'. For each trial, children heard a stimulus nonword and saw an array of four pictures. The target object was presented with three of the other trained objects in a $2 \times 2$ grid. The position of the target was counterbalanced and each picture was used as a distracter for an equal number of trials. Children were required to select the target using a key press. The keys Q, W, A and S on a standard keyboard were used for this purpose and stickers were placed on these keys so that children could map them onto the $2 \times 2$ grid on the screen. Instructions and practise trials ensured that children understood the demands of the task before targets 
were presented. The E-Prime program (Schneider et al., 2002a, 2002b) controlled random presentation of trials and recorded accuracy and reaction time data.

Spelling. Children were given a piece of paper and a pencil and were instructed to spell each nonword to dictation. Nonwords were presented in a random order.

\section{Background measures}

Standardised measures of nonverbal reasoning, vocabulary and reading skills were administered prior to the experiment when children were approximately nine months younger $(M$ age $=8.26$ years, $S D=.26)$. Alongside the experiment reading skills were assessed in more detail by administering separate measures of regular word, exception word and nonword reading skills. Including background measures allowed us to investigate the relationship between performance in the word learning experiment and individual differences in cognitive skills.

\section{Nonverbal reasoning}

This was measured using the Matrix Reasoning subtest of the Wechsler Abbreviated Scale of Intelligence (WASI; Wechsler, 1999). This subtest assesses nonverbal reasoning using a pattern completion task. The WASI provides norms for individuals aged 6-89 years.

Vocabulary skills

This was measured using the Vocabulary subtest of the WASI (Wechsler, 1999). This subtest is

a measure of expressive vocabulary in which children are asked to verbally define words. The WASI provides norms for individuals aged 6-89 years.

Reading skills

Prior to the experiment, word and nonword reading skills were assessed using the Test of Word Reading Efficiency (TOWRE; Torgesen, Wagner, \& Rashotte, 1999). In this test children are asked to read a list of items of increasing length and difficulty as quickly as they can. Efficiency is indexed by the number of nonword and words read correctly in 45 seconds, to give totals for Phonemic Decoding 
Efficiency (PDE) and Sight Word Efficiency (SWE) respectively. The test provides norms for individuals aged 6-24 years.

Alongside the word learning experiment, reading skills were assessed using lists of nonwords and words from Bates et al. (2004). These experimental lists allowed us to assess separately the reading of exception words, regular words and nonwords. It was of particular interest to assess exception word reading skills as a measure of existing orthographic knowledge. Spelling-sound correspondences for regular words and nonwords are consistent. Therefore, these words can be readily decoded on the basis of grapheme-phoneme conversion rules. This is not the case for exception words (e.g., blood, eye, meringue, yacht) and arguably reading of exception words is more reliant on orthographic skills. Children were presented with a list of 40 exception words to read, followed by 40 regular words and then 40 nonwords.

\section{RESULTS}

\section{Training}

Performance in the three production sessions, where the child had to produce the spoken name of a pictured item, is presented in Figure 1. This clearly shows that learning increased over sessions. This task was quite difficult, with children correctly producing a proportion of .40 items on average by the end of training (range $=.00-.83$ items). Figure 1 also suggests that by Session 2, children learned nonwords better when orthography was present. Performance on consistent (C) and inconsistent consonant (IC) items was similar across sessions, whereas children correctly produced fewer inconsistent vowel (IV) items. 


\section{(Figure 1 about here)}

As the dependent variable for the training measure is bounded, raw scores were subjected to an angular transformation (as recommended by Kirk, 1968, p. 66). However, analyses conducted with raw scores yielded an identical pattern of results. A 3x2x3 ANOVA was conducted with session (1 vs. 2 vs. 3), orthography (absent vs. present) and consistency (C vs. IC vs. IV) as repeated measures. This yielded significant main effects of session $\left(F(2,114)=126.12, p<.001, \eta^{2}=.69\right)$, orthography $(F(1$, $\left.57)=52.05, p<.001, \eta^{2}=.48\right)$ and consistency $\left(F(2,114)=12.03, p<.001, \eta^{2}=.17\right)$. There were also significant 2-way interactions between session and orthography $\left(F(2,114)=25.49, p<.001, \eta^{2}=.31\right)$ and session and consistency $\left(F(4,228)=3.07, p=.02, \eta^{2}=.05\right)$.

Tests of simple effects (using Bonferroni correction for multiple comparisons) revealed that i) performance improved significantly with each new session, ii) the interaction between session and orthography was driven by an effect of orthography in Sessions 2 and 3, but not in Session 1, iii) the main effect of consistency reflected particular difficulty learning items in the IV condition, while C and IC conditions did not differ from each other and iv) this latter pattern was only observed in Session 2, in Sessions 1 and 3 there were no significant differences between consistency conditions.

Despite the angular transformation conducted on these data, they did not strictly meet parametric assumptions. Therefore, to support the parametric analyses described above we carried out three nonparametric tests. Although nonparametric tests cannot be used to investigate interactions between related samples variables, Wilcoxon signed-ranks tests confirmed our main claim that orthography promotes learning in Sessions $2(T=35, p<.001, r=-.50)$ and $3(T=46, p<.001, r=-.50)$ but not in Session $1(T=209, p>.05, r=-.08)$. Analyses by items were not considered to be appropriate due to the small number of items. However, the proportion of participants producing a correct response for each item by training session, orthography and condition is presented in Appendix A. In line with 
the analyses, this shows that an orthographic facilitation effect generalises across most items in Sessions 2 and 3. In sum, children's performance improved with training. Consistent with our hypothesis, the presence of orthography boosted learning of phonology-semantic links, but not in the first trial.

Before considering performance in the post-tests, it is worth noting that in the training session presentation time of stimuli was variable as it was determined by the time taken for a child to repeat the nonword. We thank an anonymous reviewer for suggesting that the effect of orthography during training could be underpinned by an overall difference in inspection time across orthography conditions. Potentially, longer inspection time during orthography present trials could have conferred a learning advantage for these trials. Data on total inspection time for each trial was available and this proposal was examined using a $t$-test to compare the grand mean processing time in orthography absent $(M=3250 \mathrm{~ms}, S D=474 \mathrm{~ms})$ and orthography present $(M=3065 \mathrm{~ms}, S D=513 \mathrm{~ms})$ trials. This difference was significant $(t=3.17, p=.002)$. However, the overall mean difference was very small (185 ms) and further, processing time was greater in the orthography absent condition. Therefore, improved performance for items trained with orthography was not due to increased processing time.

\section{Post-tests}

\section{Nonword-picture matching}

A nonword-picture matching task was used to assess learning for novel word meanings; this post-test yielded both accuracy and RT data. However, accuracy (proportion correct) was close to ceiling $(M=.82, S D=.16$, range $=.42-1.00 ; 22.4 \%$ of children were at ceiling $)$ and data were far from normally distributed. Therefore, RTs were analysed. One child's data did not record correctly so analyses were conducted with a sample of 57 children. RTs for incorrect responses were discarded (20\%). RTs were trimmed to a maximum of 2SD from each child's mean, such that RTs outside of that 
limit ( $2 \%$ of $\mathrm{RTs}$ ) were replaced with $\mathrm{M} \pm 2 \mathrm{SD}$ for that child (one of the methods recommended by Ratcliff, 1993). This method of replacing rather than removing extreme values allowed us to maximise the number of observations on which analyses were based. Any missing values (5\%) were replaced with the mean RT for that condition. A log transformation rendered data that were normally distributed for analyses. Figure 2 summarises untransformed mean RT data. Proportion accuracy is included above each bar for reference.

\section{(Figure 2 about here)}

The RT data in Figure 2 suggests that while consistency did not have an effect on overall performance, orthography boosted performance, particularly in C and IV trials. A 2x3 ANOVA was conducted with orthography (absent vs. present) and consistency (C vs. IC vs. IV) as repeated measures. This revealed a main effect of orthography $\left(F(1,56)=5.50, p=.02, \eta^{2}=.09\right)$, with reduced RTs for items that had been trained with orthography. There was also a main effect of consistency $(F(2$, $\left.112)=3.74, p=.03, \eta^{2}=.06\right)$ but the orthography by consistency interaction was not significant. Tests of simple effects revealed that the effect of consistency reflected a significant difference between performance on $\mathrm{C}$ items and $\mathrm{IC}$, with slower performance on the latter. There were no other significant differences between consistency conditions. Mean RT for correct responses by items is presented in Appendix A. Although it was not deemed appropriate to analyse these data, they show that the presence of orthography was associated with a reduction in RT for all consistent items and five out of eight inconsistent items. In line with the hypotheses set out in the Introduction, the presence of orthography during training facilitated performance on the nonword-picture matching task. 


\section{Spelling}

Spellings were scored as correct if children produced the target orthographic pattern (see Appendix A). ${ }^{1}$ These accuracy scores are presented as solid bars in Figure 3. The Figure suggests that when orthography was absent, children were able to spell C items well, but performance on items in IC and IV conditions was close to floor. The presence of orthography had little effect on spelling of C items but dramatically improved performance on IC and IV items.

(Figure 3 about here)

As the spelling measure is bounded, raw scores were subjected to an angular transformation. These data were analysed using a 2x3 ANOVA with orthography (absent vs. present) and consistency (C vs. IC vs. IV) as repeated measures. There were significant main effects of orthography $(F(1$, $\left.57)=185.09, p<.001, \eta^{2}=.77\right)$ and consistency $\left(F(2,114)=42.55, p<.001, \eta^{2}=.43\right)$ and a significant orthography $\mathrm{x}$ consistency interaction $\left(F(2,114)=30.36, p<.001, \eta^{2}=.35\right)$. Tests of simple effects (using Bonferroni correction for multiple comparisons) revealed that orthography had a significant effect on spelling in both inconsistent conditions (IC and IV), but not in the C condition. Also, when orthography was absent during training, performance for $\mathrm{C}$ items was significantly better than for inconsistent conditions, which did not differ from each other. When orthography had been present during training, there were no differences between performance in the three consistency conditions.

As with the training measure, nonparametric tests were conducted to confirm the key finding that the presence of orthography boosted spelling performance. In line with the main analyses, Wilcoxon signed-ranks tests revealed that this was the case for IC ( $T=0, p<.001, r=-.55)$ and IV ( $T$ $=22, p<.001, r=-.56)$ items but not $\mathrm{C}$ items $(T=248, p>.05, r=-.09)$. Accuracy by items is presented 
in Appendix A. Although the number of items per condition was too small for statistical analysis, the data show that the presence of orthography was associated with improved spelling performance across all inconsistent items and three out of four consistent items. In sum, even though children's attention was not directed to orthography, its presence during training was associated with improved orthographic learning. Performance on $\mathrm{C}$ items was generally high, indicating that children could accurately infer their spelling patterns from phonology alone. When orthography had been present during training, this additional information boosted spelling, resulting in performance on IC and IV items that was equivalent to performance on $\mathrm{C}$ items.

\section{Error analysis for the spelling data}

For each inconsistent item, one spelling pattern was adopted as being 'correct'. However, by definition each item has more than one legal spelling. Therefore, children could make 'errors' that corresponded to plausible spellings. To investigate the overall plausibility of the spelling patterns that children produced, performance was re-scored. The dotted bars in Figure 3 show accuracy when all plausible spellings are accepted as correct. Note that performance in the $\mathrm{C}$ condition remains unchanged by this adjustment. The dotted bars indicate that children produced many plausible spellings for the inconsistent nonwords, even when they had not been exposed to orthography. This suggests that these children could successfully translate phonological information into appropriate orthographic strings. When orthography had been present at training, some children produced the alternative legal spelling patterns rather than the target orthography (represented by the gap between solid and dotted bars). This suggests that in some cases children had not acquired word-specific orthography during training, but rather they were translating (incorrectly) from phonology during the spelling post-test. We did not direct children's attention to orthography during training, and doing so might result in more children learning word-specific orthography. 


\section{Individual differences}

Table 1 summarises performance on background measures of nonverbal reasoning, vocabulary and reading ability. Mean standard scores for this sample are close to population norms. Further, on experimental reading lists children obtained high scores when reading regular words and lower scores when reading exception words and nonwords.

(Table 1 about here)

Orthographic facilitation (OF) scores for training, nonword-picture matching and spelling measures were calculated to capture the degree to which children benefited from orthography. These scores are presented in Table 2. OF scores for training and spelling were calculated using log odds to account for floor and ceiling effects (Allerup \& Elbro, 1998) and scores for the nonword-picture matching task were derived by subtracting between RTs. For all, greater magnitude corresponds to more facilitation. OF scores are not reported for the first training session as the presence of orthography did not have a significant effect until Session 2.

(Table 2 about here)

Previous research using similar paradigms has shown that more advanced readers show superior learning overall and more benefit from orthography during training (Ehri \& Wilce, 1979; Rosenthal \& Ehri, 2008). Calculating an OF score allowed us to explore the more specific hypothesis that better scores on reading (and other background measures) would be correlated with the degree to which 
children benefit from the presence of orthography. No significant correlations between background measures and $\mathrm{OF}$ in the training phase were observed, however, correlations were observed with performance on nonword-picture matching and spelling post-tests. The results of these analyses will be discussed for each post-test in turn.

In the nonword-picture matching task the RT data for one participant did not record properly, therefore the OF score was calculated for 57 children. OF was not correlated with age, nonverbal reasoning and vocabulary skills (all $p \mathrm{~s}>.05$ ). OF was marginally correlated with nonword reading as measured by the TOWRE PDE subtest $(r(57)=.25, p=.06)$, but not with the experimental measure of nonword reading. Higher correlations were observed between $\mathrm{OF}$ and word reading measures; OF was significantly correlated with TOWRE word reading (SWE subtest: $r(57)=.30, p<.05$ ), regular word reading $(r(57)=.30, p<.05)$ and exception word reading $(r(57)=.38, p<.01)$. In sum, children with better word reading accuracy scores showed more benefit from orthography. The strongest correlation was with exception word reading, arguably a purer measure of orthographic processing skill. However, $t$ tests showed that none of the differences between the three correlation coefficients were significant (all ps>.05).

As OF scores for spelling were not normally distributed, nonparametric correlations are reported. OF was not correlated with age, nonverbal reasoning and vocabulary skills (all $p \mathrm{~s}>.05$ ). However, OF was significantly correlated with nonword reading (TOWRE PDE: $r(58)=.28, p<.05$; experimental nonwords: $r(58)=.36, p<.01)$, TOWRE SWE $(r(58)=.34, p<.01)$, experimental regular words $(r(58)=.26, p<.05)$ and experimental exception words $(r(58)=.29, p<.05)$. Again $t$-tests showed that the differences between correlation coefficients were not significant (all $p \mathrm{~s}>.05$ ). Overall, more advanced readers showed more benefit from orthography in the spelling task. 


\section{DISCUSSION}

The primary aim of this study was to investigate whether the incidental presence of orthography during training would improve learning of oral vocabulary. To approximate vocabulary acquisition, we used a learning paradigm to investigate learning of nonword-referent (verbal-visual) pairings. Children were more able to produce target spelling patterns for items that had been trained with orthography, even though they had not been alerted to its presence, nor had they been instructed to use it. In addition, the presence of orthography facilitated learning for nonword-referent pairings both during training and at post-test. We will discuss the role of orthography during training and in post-tests, and then consider our secondary aim, the relationship between individual differences in orthographic processing skill and orthographic facilitation.

Performance in the production sessions of the training phase and in the nonword-picture matching post-test reflect learning of phonology, referents (semantics) and links between the two. By the end of training average performance was relatively low, suggesting that producing phonology in response to the referents was difficult. During training and at post-test, learning was significantly improved for consistent and inconsistent items trained with orthography. Furthermore, little exposure to orthography was needed for it to have a powerful effect on learning. By Session 2 of training, when presented with a novel object, children were more likely to produce its name if it had been paired with orthography previously. This is consistent with previous studies that show that children are more likely to learn phonological forms when they are presented with visual or orthographic information (e.g., Ehri \& Wilce, 1979; Hu, 2008; Hulme et al., 2007; Reitsma, 1983). Further, we interpret this finding as demonstrating that learning is improved for word representations that include phonological, semantic and orthographic information (cf. the Lexical Quality Hypothesis; Perfetti \& Hart, 2002). Although our study was not designed as a replication of Rosenthal and Ehri (2008), using somewhat different methodology, the two studies converge on the finding that the presence of orthography facilitates oral 
vocabulary acquisition in children. However, it is important to note that in Rosenthal and Ehri's study children learned to associate new labels with definitions that were closely related to known concepts. In our study, although children learned novel information, semantics was represented using pictures of novel objects. Further, in both studies children demonstrated semantic learning using a relatively simple response. By instructing children that the objects were things that an alien would use and since adults attributed a function or category to the objects (see method section) we hoped to ensure that children were learning semantics. However, it is clear that a referent provides a limited semantic representation. The finding that orthography facilitates learning for new word meaning needs to be replicated in a paradigm that elicits richer and genuinely novel semantic representations, for instance, by teaching children definitions for novel words. Therefore, an important future step would be to investigate whether the presence of orthography helps children to acquire and retain more complex semantic information about new words.

In naturalistic oral vocabulary acquisition, not only is semantic information likely to be more complex, but once a word is integrated into the lexicon, representations will include rich connections with other words. Some studies have addressed this issue in adults (e.g., Gaskell \& Dumay, 2003), showing that learned words interact with the processing of similar known words. An interesting direction for future research would be to adapt our methodology to explore children's integration of words within the lexicon. This would allow investigation of whether learned words are incorporated into a child's semantic network, and how this impacts on the processing of other words.

An important question that arises from this research (see also Rosenthal \& Ehri, 2008) is whether the purported effect of orthography on semantic learning is mediated entirely by the relationship between orthography and phonology. It is likely that in this experiment orthography aided the acquisition of phonological representations. A number of researchers have argued that orthography can aid learning and retention of phonological information (e.g., Ehri, 2005; McKague et al., 2008; Hu, 2008). This effect may have underpinned the apparent interaction between orthography and semantics. 
Alternatively, orthography may have exerted an influence on semantic learning more directly. This is of interest because models of reading (e.g., Harm \& Seidenberg, 2004) emphasise direct links between orthography and semantics. The design of this experiment did not allow us to tease apart these two possible interpretations. A next step in this line of research would be to design experiments that systematically assess how phonology mediates the orthographic facilitation effect on semantic learning.

Orthographic learning was assessed with a spelling post-test. Children produced many target spellings for consistent items, irrespective of the presence or absence of orthography. This is unsurprising as our participants had experienced approximately five years of literacy instruction and these stimuli were constructed so that orthography could be reliably predicted from phonology. The error analysis suggested that when children produced incorrect spelling patterns for inconsistent items, many were plausible on the basis of phoneme-grapheme mappings. These results suggest that children's knowledge of phoneme $\rightarrow$ grapheme relations was sufficient to produce appropriate spelling patterns for many of the items without prior exposure to orthography. While the presence of orthography during training did not affect spelling of consistent items, it dramatically improved spelling of inconsistent items, resulting in performance that was equivalent to that for consistent stimuli (cf. Rosenthal \& Ehri, 2008). In line with Share's self-teaching hypothesis (Share, 1995), incidental exposure to orthography led children to encode word-specific orthographic representations for many of the items.

As noted in the Introduction, consistency was manipulated in this experiment to simulate the complexity of English orthography and to investigate orthographic learning for items where orthography cannot be readily inferred from phonology. In the case of inconsistent consonant items this was done by using phonemes that can be represented by consonant clusters that include a silent letter this source of sound to spelling inconsistency occurs in many English words (e.g., limb, knife, batch, write). Vowel consistency was calculated using type and token frequency data from the Children's Printed Word Database (Masterson et al., 2002). When considered in isolation, this data confirmed that 
vowels were either consistent (consistent and inconsistent consonant item sets) or inconsistent (inconsistent vowel set) in terms of the frequency with which the vowel phoneme is spelled in a particular way. However, the consistency of a vowel spelling is also influenced by the context in which it appears, for instance, the consonants that make up the rime in syllables (for a detailed discussion of this issue see Kessler \& Treiman, 2001; Caravolas, Kessler, Hulme, \& Snowling, 2005). Furthermore, we did not control for orthographic legality or phonological complexity of items, and post hoc analyses indicated these factors could have made the inconsistent items more difficult. Potential effects of uncontrolled variables are inherent in studies that compare performance on lists of verbal stimuli (e.g., Bowers, Davis, \& Hanley, 2005). This highlights the need for future orthographic learning studies to consider consistency and variables confounded with consistency in detail (for examples of where this has been done with adults see Burt \& Blackwell, 2008; McKague et al., 2008; McKay, Davis, Savage, \& Castles, 2008).

We turn now to discuss the relationship between individual differences in reading (and other cognitive measures) and the degree of orthographic facilitation observed for each learning measure. Rosenthal and Ehri's (2008) study provided evidence that better readers show more benefit from orthography. Specifically, they showed that reading level in US children from low socio-economic and ethnic minority backgrounds was associated with pronunciation and spelling measures, and with some variables tapping learning for meaning. In our study, we recruited children from UK schools serving mixed social catchment areas. Therefore, our sample may reflect greater reading variation. Individual differences were not related to orthographic facilitation during training. However, the amount of orthographic facilitation observed in spelling and nonword-picture matching tasks was associated with reading level. More advanced readers, who presumably have more experience with, and knowledge of orthography, showed greater benefit from the presence of orthography. We assessed exception word, regular word and nonword reading separately as well as including standardised measures of nonword reading and word reading. Using a similar learning paradigm, Hulme et al. (2007) found that learning 
was more closely related to exception word reading than nonword reading. In addition, exception word reading is thought to be a relatively pure measure of orthographic processing skill (although see Burt, 2006; Castles \& Nation, 2006) and as such might have been more closely related to orthographic facilitation than other measures of reading. However, this was not borne out by $t$-tests comparing the significance of correlation coefficients.

The finding that better readers benefit more from orthography in word learning paradigms has potential implications for education and intervention. Overall, teaching new words with orthography might be beneficial in mainstream classrooms. Further, presenting orthography could provide a compensatory strategy for children who find it difficult to learn new words, whilst having relatively good visual or orthographic skills. For instance, there is evidence that a pattern of relative strengths in reading or orthography alongside weaknesses in phonology and/or vocabulary can be observed in some children and adults with Down's syndrome (e.g., Cossu, Rossini, \& Marshall, 1993; Laws \& Gunn, 2002; Roch \& Jarrold, 2008; Roch \& Levorato, in press). A similar dissociation between orthographic and semantic skills is also characteristic of poor comprehenders, children with age-appropriate word recognition skills alongside comprehension weaknesses (Ricketts, Bishop, \& Nation, 2008). In contrast, our results suggest that teaching new words with orthography is less likely to benefit children with poor word recognition skills (i.e., in dyslexia). However, Rosenthal and Ehri (2008) found that even poor readers benefited from orthography when learning new words. The presence of orthography was incidental in both their study and ours. Potentially, poor readers would show more benefit from orthography if its presence were made more explicit in the learning task. The proposal that orthography might be used to support vocabulary learning in children with reading and language weaknesses warrants further investigation.

Before concluding, it is worth noting two general limitations of the study. First, children learned only 12 stimuli to ensure that training and post-tests could be completed in one testing session. This meant that scores for items in each consistency condition, within each orthography condition were 
based on performance on only two items. In future research we would aim to replicate and extend our findings in a paradigm with greater statistical power. This could be done by examining the role of orthography without a consistency manipulation, by using a between subjects design or simply by increasing the number of items by conducting the experiment over a number of testing sessions. Also, it would be important to see the extent to which our findings are replicated in analyses by items. Performance by item is reported in Appendix A. However, items analyses were not deemed appropriate with such a small number of items.

The second limitation concerns our interpretation of the findings. In line with a previous study (Rosenthal \& Ehri, 2008) we suggest that improved performance in the orthography present condition reflects facilitation from orthography. In the orthography absent condition, children were trained on two sources of information (phonology and semantics) whereas in the orthography present condition, children were presented with three (phonology, semantics and orthography). It is possible that the purported effect of orthography is not specific to orthography and instead reflects a simpler effect of exposure to more information. This alternative hypothesis could be assessed by extending the current paradigm to include additional conditions in which children are exposed to three sources of information but the third representation is not orthography (e.g., abstract symbols). Although this limitation is of theoretical importance, practically it appears that vocabulary acquisition is improved in conditions where orthography is present. This finding should be incorporated into approaches to vocabulary instruction that do not currently emphasise a role for orthography (e.g., Beck et al., 2002).

In conclusion, this study was primarily designed to investigate whether the presence of orthography would aid learning of new vocabulary. Even though children were not alerted to the presence of orthography or instructed to use it, nonwords were more likely to be learned if they had been trained with orthography. Also, children with better reading skills were more likely to benefit from orthography. Prior exposure to orthography was selectively beneficial to orthographic learning for inconsistent items. This provides clear support for the self-teaching hypothesis (Share, 1995) in 
demonstrating that when orthography is available to children, they use it to produce target spelling patterns. While this result is relatively intuitive, more striking is the finding that presenting orthography in an incidental manner seemed to strengthen links between phonology and semantics, as shown during training and in the nonword-picture matching task. Thus, it appears that in addition to supporting phonological representations (e.g., Ehri, 2005; Ehri \& Wilce, 1979; Hu, 2008), orthography may serve to support the acquisition of new word meanings. Future studies should aim to replicate this finding in studies that allow children to acquire novel and complex semantic information. Further, studies are needed to explore the mechanisms via which orthography supports the development of phonological and/or semantic representations. However, our study converges with previous work (Rosenthal \& Ehri, 2008) in suggesting that children will benefit from teaching methods that present new vocabulary with orthography, especially if they have good existing knowledge of orthography. Together, these studies provide an evidence base for the practice of emphasising orthography when teaching new words in the classroom. 


\section{References}

Allerup, P., \& Elbro, C. (1998). Comparing differences in accuracy across conditions or individuals: An argument for the use of log odds. Quarterly Journal of Experimental Psychology, 51A, 409424.

Bates, T. C., Castles, A., Coltheart, M., Gillespie, N., Wright, M., \& Martin, N. G. (2004). Behaviour genetic analyses of reading and spelling: A component processes approach. Australian Journal of Psychology, 56, 115-126.

Beck, I., McKeown, M., \& Kucan, L. (2002). Bringing words to life: Robust vocabulary instruction. New York: Guildford Press.

Bowers, J., Davis, C., \& Hanley, D. (2005). Automatic semantic activation of embedded words: Is there a "hat" in "that"? Journal of Memory and Language, 52(1), 131-143.

Bowey, J. A., \& Miller, R. (2007). Correlates of orthographic learning in third-grade children's silent reading. Journal of Research in Reading, 30, 115-128.

Burt, J. S. (2006). What is orthographic processing skill and how does it relate to word identification in reading? Journal of Research in Reading, 29, 400-417.

Burt, J. S., \& Blackwell, P. (2008). Sound-spelling consistency in adults' orthographic learning. Journal of Research in Reading, 31, 77-96.

Caravolas, M., Kessler, B., Hulme, C., \& Snowling, M. (2005). Effects of orthographic consistency, frequency, and letter knowledge on children's vowel spelling development. Journal of Experimental Child Psychology, 92, 307-321.

Castles, A., Holmes, V., Neath, J., \& Kinoshita, S. (2003). How does orthographic knowledge influence performance on phonological awareness tasks? Quarterly Journal of Experimental Psychology Section a-Human Experimental Psychology, 56, 445-467. 
Castles, A., \& Nation, K. (2006). How does orthographic learning happen? In S. Andrews (Ed.), From inkmarks to ideas: Challenges and controversies about word recognition and reading (pp. 151179). Hove, East Sussex: Psychology Press.

Cossu, G., Rossini, F., \& Marshall, J. (1993). When reading is acquired but phonemic awareness is not: a study of literacy in Down's syndrome. Cognition, 46, 129-138.

Cunningham, A. E. (2006). Accounting for children's orthographic learning while reading text: Do children self-teach? Journal of Experimental Child Psychology, 95, 56-77.

Ehri, L. C. (2005). Development of sight word reading: Phases and findings. In M. Snowling \& C. Hulme (Eds.), The science of reading: A handbook (pp. 135-154). Oxford, England: Blackwell.

Ehri, L. C., \& Wilce, L. (1979). The mnemonic value of orthography among beginning readers. Journal of Educational Psychology, 71, 26-40.

Gaskell, M. G., \& Dumay, N. (2003). Lexical competition and the acquisition of novel words. Cognition, 89, 105-132.

Harm, M., \& Seidenberg, M. S. (2004). Computing the meanings of words in reading: Cooperative division of labor between visual and phonological processes. Psychological Review, 111, 662720.

Hu, C. F. (2008). Use orthography in L2 auditory word learning: Who benefits? Reading and Writing, $21,823-841$.

Hulme, C., Goetz, K., Gooch, D., Adams, J., \& Snowling, M. J. (2007). Paired-associate learning, phoneme awareness, and learning to read. Journal of Experimental Child Psychology, 96, 150166.

Kessler, B., \& Treiman, R. (2001). Relationships between sounds and letters in English monosyllables. Journal of Memory and Language, 44, 592-617.

Kirk, R. (1968). Experimental design: Procedures for the behavioural sciences. Belmont, CA: Brooks/Cole. 
Laws, G., \& Gunn, D. (2002). Relationships between reading, phonological skills and language development in individuals with Down syndrome: A five year follow-up study. Reading and Writing, 15, 527-548.

Masterson, J., Dixon, M. \& Stuart, M. (2002). The Children's Printed Word Database, Retrieved 8 November 2005 from http://www.essex.ac.uk/psychology/cpwd/

McKague, M., Davis, C., Pratt, C., \& Johnston, M. B. (2008). The role of feedback from phonology to orthography in orthographic learning: An extension of item-based accounts. Journal of Research in Reading, 31, 55-76.

McKay, A., Davis, C., Savage, G., \& Castles, A. (2008). Semantic involvement in reading aloud: Evidence from a nonword training study. Journal of Experimental Psychology: Learning, Memory, and Cognition, 34, 1495-1517.

Nation, K., Angell, P., \& Castles, A. (2007). Orthographic learning via self-teaching in children learning to read English: effects of exposure, durability and context. Journal of Experimental Child Psychology, 96, 71-84.

Nelson, J. R., Balass, M., \& Perfetti, C. A. (2005). Differences between written and spoken input in learning new words. Written Language \& Literacy, 8, 25-44.

Perfetti, C. A., Bell, L. C., \& Delaney, S. M. (1988). Automatic (prelexical) phonetic activation in silent word reading: Evidence from backward masking. Journal of Memory and Language, 27, 59-70.

Perfetti, C. A., \& Hart, L. (2002). The lexical quality hypothesis. In L. Verhoeven, C. Elbro \& P. Reitsma (Eds.), Precursors of functional literacy (Vol. 11). Amsterdam/Philadelphia: John Benjamins Publishing Company.

Ratcliff, R. (1993). Methods for dealing with reaction time outliers. Psychological Bulletin, 114, 510532. 
Reitsma, P. (1983). Printed word learning in beginning readers. Journal of Experimental Child Psychology, 36(2), 321-339.

Ricketts, J., Bishop, D. V. M., \& Nation, K. (2008). Investigating orthographic and semantic aspects of word learning in poor comprehenders. Journal of Research in Reading, 31, 117-135.

Roch, M., \& Jarrold, C. (2008). A comparison between word and nonword reading in Down syndrome: The role of phonological awareness. Journal of Communication Disorders, 41, 305-318.

Roch, M., \& Levorato, C. M. (in press). Simple View of Reading in Down's syndrome: The role of listening comprehension and reading skills. International Journal of Language \& Communication Disorders.

Rosenthal, J., \& Ehri, L. C. (2008). The mnemonic value of orthography for vocabulary learning. Journal of Educational Psychology, 100, 175-191.

Schneider, W., Eschman, A., \& Zuccolotto, A. (2002a). E-Prime reference guide. Pittsburgh: Psychology Software Tools Inc.

Schneider, W., Eschman, A., \& Zuccolotto, A. (2002b). E-Prime user's guide. Pittsburgh: Psychology Software Tools Inc.

Seidenberg, M. S., \& Tanenhaus, M. K. (1979). Orthographic effects on rhyme monitoring. Journal of Experimental Psychology: Human Learning and Memory, 5, 546-554.

Share, D. L. (1995). Phonological recoding and self-teaching: Sine qua non of reading acquisition. Cognition, 55, 151-218.

Share, D. L. (2004). Orthographic learning at a glance: On the time course and developmental onset of self-teaching. Journal of Experimental Child Psychology, 87, 267-298.

Torgesen, J., Wagner, R., \& Rashotte, C. (1999). Test of Word Reading Efficiency. Austin, TX: Pro-Ed. Ventura, P., Morais, J., \& Kolinsky, R. (2007). The development of the orthographic consistency effect in speech recognition: From sublexical to lexical involvement. Cognition, 105, 547-576. 
Ventura, P., Morais, J., Pattamadilok, C., \& Kolinsky, R. (2004). The locus of the orthographic consistency effect in auditory word recognition. Language and Cognitive Processes, 19, 57-95.

Wechsler, D. (1999). Wechsler Abbreviated Scale of Intelligence. London: The Psychological Corporation.

Ziegler, J. C., Jacobs, A. M., \& Klüppel, D. (2001). Pseudohomophone effects in lexical decision: Still a challenge for current word recognition models. Journal of Experimental Psychology: Human Perception and Performance, 27, 547-559. 
Appendix A. Experimental stimuli and performance by items

\begin{tabular}{|c|c|c|c|c|c|c|c|c|c|c|c|c|}
\hline \multirow{2}{*}{ Condition } & \multirow{2}{*}{ Phonology } & \multirow{2}{*}{ Orthography } & \multicolumn{6}{|c|}{ Training $^{1}$} & & & & \\
\hline & & & \multicolumn{2}{|c|}{ Session 1} & \multicolumn{2}{|c|}{ Session 2} & \multicolumn{2}{|c|}{ Session 3} & \multicolumn{2}{|c|}{ Nonword-picture matching ${ }^{2}$} & \multicolumn{2}{|c|}{ Spelling $^{1}$} \\
\hline \multicolumn{13}{|c|}{ Consistent (C) } \\
\hline & /bilp/ & bilp & 0.14 & 0.03 & 0.11 & 0.27 & 0.18 & 0.47 & 3810 & 3048 & 0.79 & 0.57 \\
\hline & /gal/ & garl & 0.00 & 0.07 & 0.00 & 0.30 & 0.25 & 0.43 & 3841 & 3216 & 0.25 & 0.47 \\
\hline & $/ \mathrm{l}_{\Lambda \mathrm{s}} /$ & luss & 0.13 & 0.36 & 0.57 & 0.86 & 0.63 & 0.96 & 1791 & 1311 & 0.40 & 1.00 \\
\hline & /nab/ & knarb & 0.07 & 0.04 & 0.13 & 0.25 & 0.37 & 0.43 & 3288 & 3545 & 0.30 & 0.68 \\
\hline & /roid/ & wroid & 0.03 & 0.00 & 0.13 & 0.29 & 0.23 & 0.50 & 3200 & 3087 & 0.00 & 0.61 \\
\hline & /gnt $\int /$ & gutch & 0.21 & 0.07 & 0.36 & 0.60 & 0.61 & 0.53 & 2622 & 2361 & 0.04 & 0.67 \\
\hline & /pim/ & pimb & 0.07 & 0.03 & 0.25 & 0.37 & 0.32 & 0.57 & 2597 & 2816 & 0.00 & 0.57 \\
\hline
\end{tabular}

Inconsistent

vowel (IV) 


$\begin{array}{llllllllllll}\text { /beip/ } & \text { baip } & 0.00 & 0.00 & 0.10 & 0.04 & 0.20 & 0.32 & 2790 & 2798 & 0.03 & 0.54 \\ \text { /davf/ } & \text { dowf } & 0.03 & 0.00 & 0.07 & 0.21 & 0.10 & 0.43 & 2957 & 2918 & 0.07 & 0.61 \\ \text { /jit/ } & \text { jeet } & 0.07 & 0.10 & 0.18 & 0.43 & 0.14 & 0.73 & 2629 & 1960 & 0.14 & 0.90 \\ \text { /lug/ } & \text { loog } & 0.00 & 0.13 & 0.07 & 0.17 & 0.18 & 0.33 & 2906 & 2512 & 0.21 & 0.83\end{array}$

Note. O- = orthography absent; O+ = orthography present; ${ }^{\mathrm{I}}$ Proportion of participants producing an accurate response; ${ }^{2} \mathrm{Mean} \mathrm{RT}(\mathrm{ms})$ to produce correct response 
Appendix B. Example of nonword-referent pairing in orthography absent and present conditions

Orthography absent

$\underline{\text { Hear: }}$

See:

$$
\text { /dauf/ }
$$

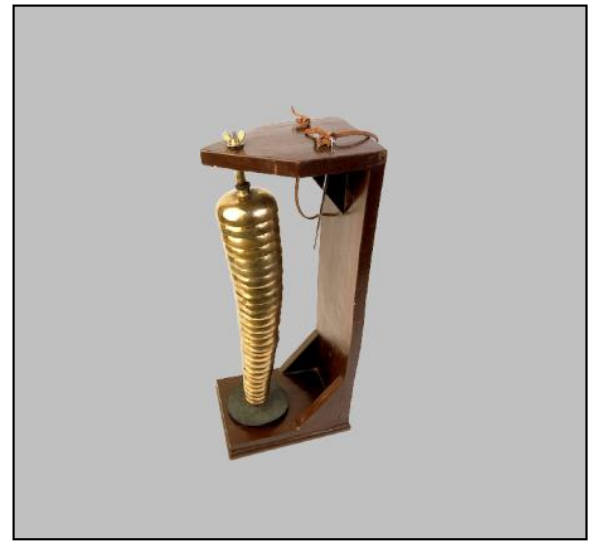

Orthography present

$$
\text { /dauf/ }
$$

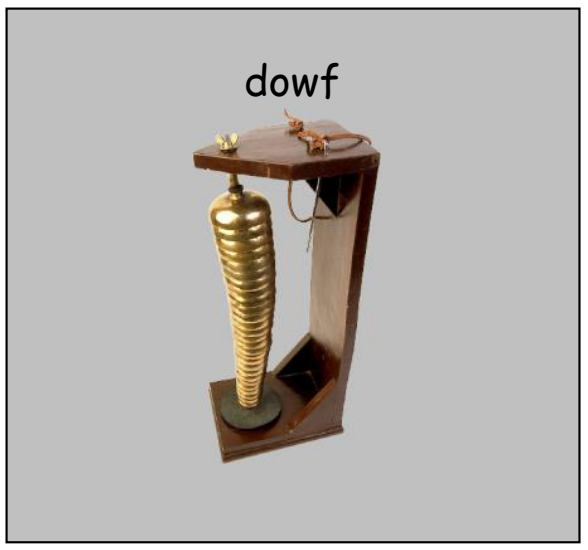




\section{Author notes}

Jessie Ricketts, D.V.M. Bishop, Kate Nation, University of Oxford. We would like to thank The Economic and Social Research Council for a studentship awarded to the first author and grant awarded to the second and third authors. D.V.M Bishop is supported by The Wellcome Trust. We would also like to thank all teachers, parents and children for their ongoing support with our research.

Correspondence should be addressed to Jessie Ricketts, Department of Experimental Psychology, University of Oxford, South Parks Road, Oxford, OX1 3UD, UK. Email: jessie.ricketts@psy.ox.ac.uk; Web: www.psy.ox.ac.uk/lcd, www.psy.ox.ac.uk/oscci 


\section{Footnote}

${ }^{1}$ There were two exceptions to this. For two of the consistent items (luss, joig) children produced alternative but plausible spellings (lus, joyg). This was unexpected for joig, however, we added an extra s to luss to make this item longer and therefore more similar to other items. We accounted for this inconsistency by accepting these alternative spellings as correct if children had not been exposed to nonword orthography. 
Table 1.

Means and standard deviations for background measures.

Task $M$

$S D$

Standardised tests

Nonverbal reasoning ${ }^{1}$

106.00

12.14

Vocabulary $^{1}$

92.47

18.23

TOWRE Nonword reading $(\mathrm{PDE})^{1}$

101.34

14.14

TOWRE Word reading $(\mathrm{SWE})^{1}$

102.16

13.42

Experimental reading lists

Exception word reading ${ }^{2}$

.51

.11

Regular word reading ${ }^{2}$

.81

.16

Nonword reading ${ }^{2}$

.60

.23

Notes. TOWRE = Test of Word Reading Efficiency; PDE = Phonemic Decoding Efficiency; SWE =

Sight Word Efficiency; ${ }^{1}$ Standard scores; $M=100, S D=15 ;{ }^{2}$ Proportion correct 
Table 2.

Means and standard deviations for orthographic facilitation scores.

\begin{tabular}{lll}
\hline Task & $M$ & $S D$ \\
\hline Training Session 2 & & \\
${\text { Training Session } 3^{1}}^{1}$ & 1.04 & 1.18 \\
Nonword-picture matching $^{2}$ & 1.16 & 1.24 \\
Spelling $^{1}$ & 246.83 & 938.08 \\
\hline
\end{tabular}

Notes. ${ }^{1} \log$ odds score $=\ln \left[\frac{\mathrm{C}_{\text {present }}}{1-\mathrm{C}_{\text {present }}} / \frac{\mathrm{C}_{\text {absent }}}{1-\mathrm{C}_{\text {absent }}}\right]$, where $\mathrm{C}_{\text {present }}=$ proportion of correct responses for each child when orthography was present and $\mathrm{C}_{\mathrm{absent}}=$ proportion of correct responses for each child when it was absent (formula derived from Allerup \& Elbro, 1998); ${ }^{2} \Delta \mathrm{RT}$ in ms (M RT orthography absent $-M$ RT orthography present) 


\section{Figure Captions}

Figure 1. Mean proportion $( \pm \mathrm{SE})$ of nonwords learned in production training sessions across orthography and consistency conditions $(\mathrm{C}=$ consistent, $\mathrm{IC}=$ inconsistent consonant, $\mathrm{IV}=$ inconsistent vowel).

Figure 2. Mean reaction times $( \pm \mathrm{SE})$ in the nonword-picture matching task across orthography and consistency conditions $(\mathrm{C}=$ consistent, $\mathrm{IC}=$ inconsistent consonant, $\mathrm{IV}=$ inconsistent vowel $)$. Proportions above bars reflect accuracy.

Figure 3. Mean proportion correct $( \pm \mathrm{SE})$ in the spelling task across orthography and consistency conditions $(\mathrm{C}=$ consistent, $\mathrm{IC}=$ inconsistent consonant, $\mathrm{IV}=$ inconsistent vowel). Dotted bars reflect the total number of legal spelling patterns produced. 


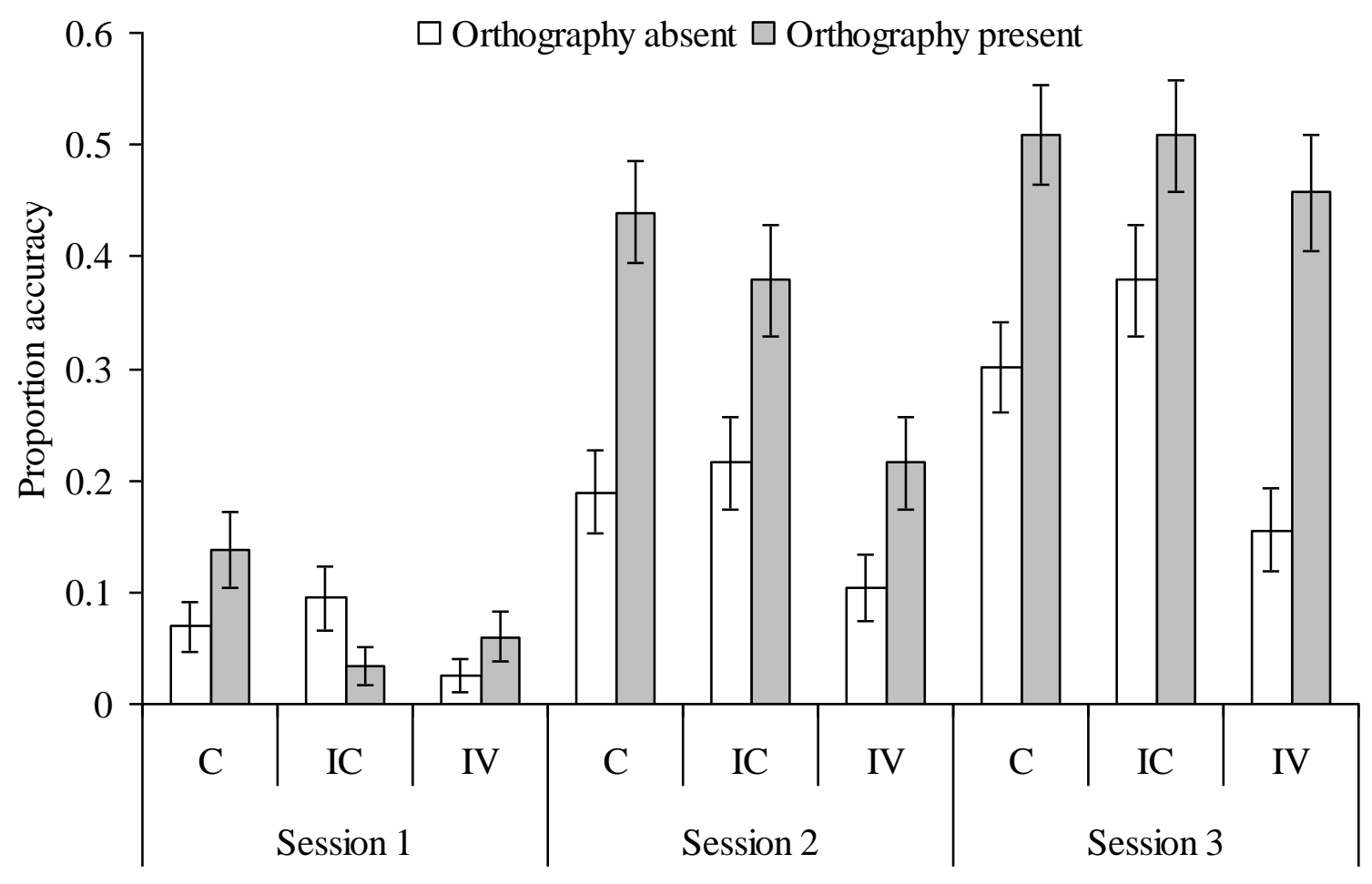




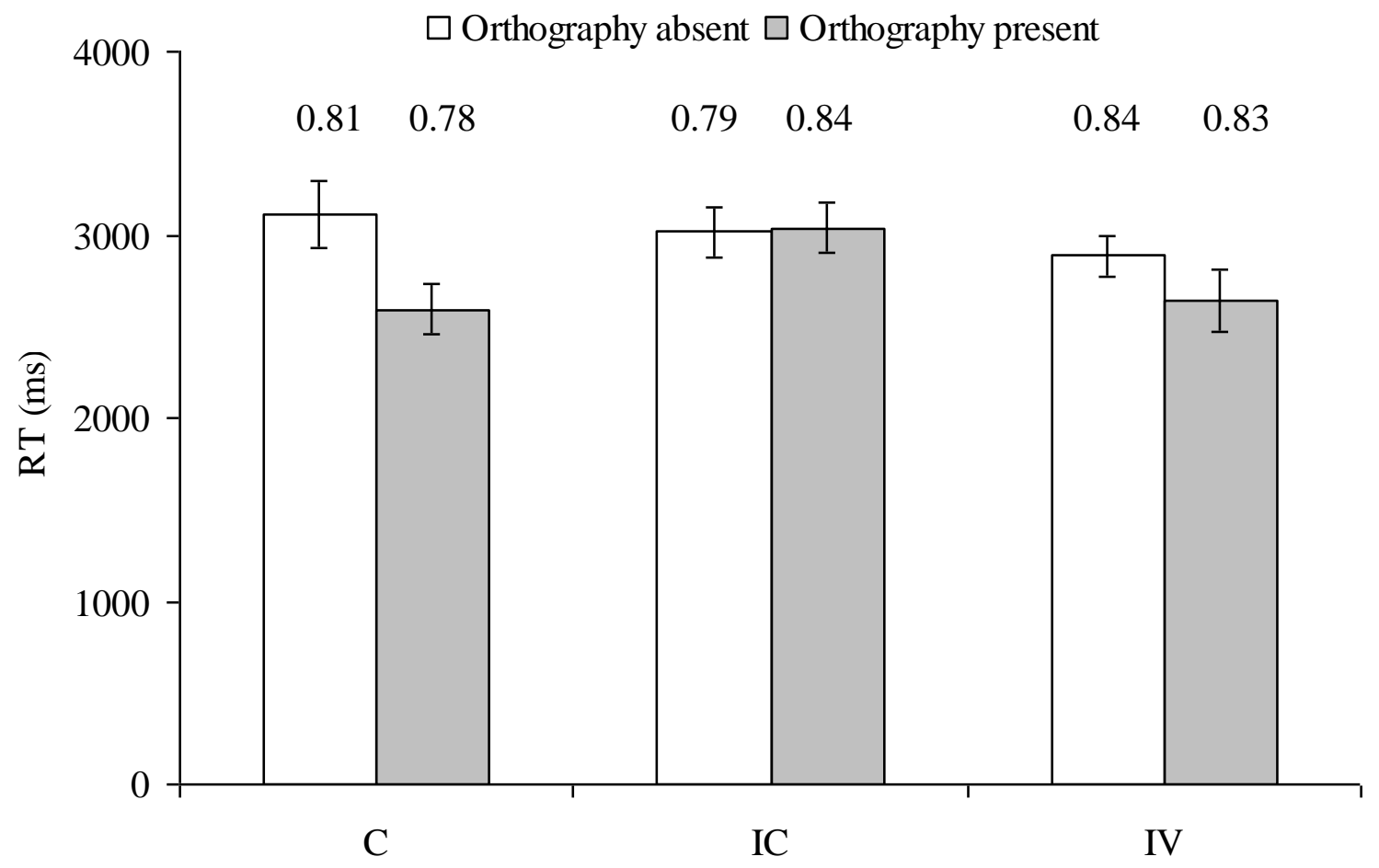




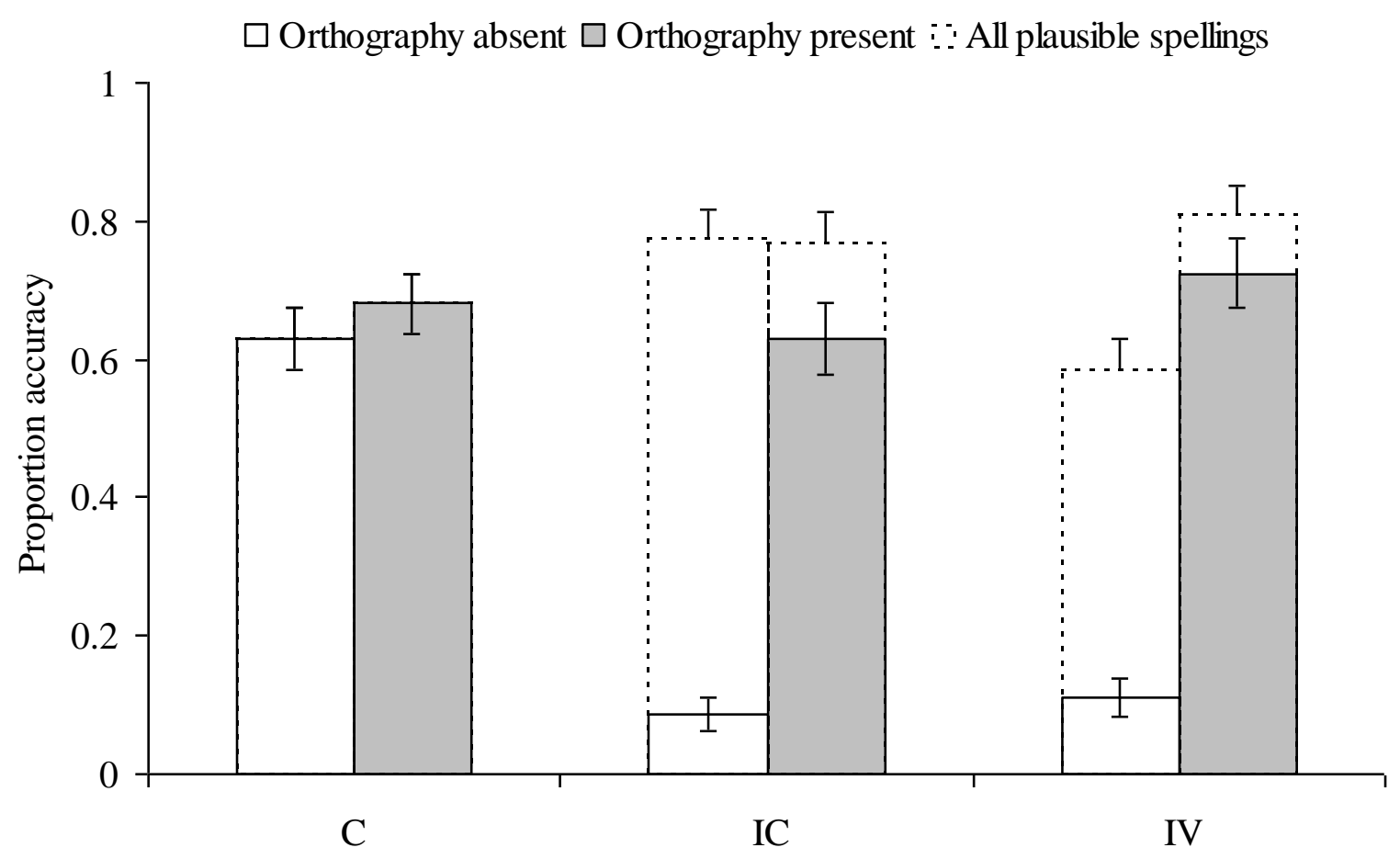

